\title{
A statistical analysis of medium-scale traveling ionospheric disturbances during 2014-2017 using the Hong Kong CORS network
}

\author{
Guanyi Chen, Chen Zhou* ${ }^{*}$, Yi Liu, Jiaqi Zhao, Qiong Tang, Xiang Wang and Zhengyu Zhao
}

\begin{abstract}
We report a statistical study of medium-scale traveling ionospheric disturbances (MSTIDs) during 2014-2017 in the low-latitude China region using the Hong Kong Continuously Operating Reference Stations network with a baseline length of 10-15 km. Polynomial fitting and the multichannel maximum entropy method are utilized to derive MSTID parameters from global positioning system total electron content data. We find that MSTIDs can be sorted into three types in this region. One type is daytime MSTIDs, which are mainly distributed during 1000-1700 local time (LT) in spring, autumn and winter. The second type is nighttime MSTIDs, which mainly occur from 2200 to 0300 LT in spring and summer. The third type is morning MSTIDs, mainly occurring from 0500 to 0700 LT in spring and autumn. No clear difference in the MSTID parameters is distinguishable between the cyclone period and non-cyclone period, which may be due to the distance restriction for cyclone identification and different propagation distances of the primary gravity waves (GWs) and secondary GWs.
\end{abstract}

Keywords: MSTID, CORS, TEC

\section{Introduction}

Medium-scale traveling ionospheric disturbances (MSTIDs) are typical wavelike perturbations of ionospheric plasma with wavelengths of hundreds of $\mathrm{kms}$, phase velocities of hundreds of $\mathrm{m} / \mathrm{s}$ and periods of between $10 \mathrm{~min}$ and $1 \mathrm{~h}$ (Hunsucker 1982). MSTIDs have been studied for many years using various techniques, such as HF Doppler radars (Waldock and Jones 1986; Oinats et al. 2016), ionosondes (Bowman 1990), satellite beacons (Jacobson et al. 1995), incoherent scatter radars (Oliver et al. 1997), ground-based Global Navigation Satellite System (GNSS) networks (Morton and Essex 1978), and airglow imagers (Shiokawa et al. 2003; Huang et al. 2016).

Several potential excitation mechanisms of midlatitude MSTIDs have been proposed in previous studies.

*Correspondence: chenzhou@whu.edu.cn

lonosphere Laboratory, School of Electronic Information, Wuhan University, Wuhan, China
TIDs have been intuitively thought to be plasma manifestations of atmospheric gravity waves (AGWs) propagating in the thermosphere (Hines 1960; Hooke 1968; Hunsucker 1982; Kirchengast et al. 1996). Based on the research of Hines, Klostermeyer (1972) supplied a full-wave solution of gravity wave propagation in a thermospheric model and compared it with the data observed from ionograms. Kirchengast et al. (1996) found the relationship between TIDs and AGWs through theoretical research and numerical simulation. Xiao et al. (2007) suggested that during the existence of a typhoon, which is one of the important ground sources of wavelike disturbances in the troposphere, there are almost always MSTIDs in the ionosphere. Kotake et al. (2007) noted that MSTIDs around the dusk sector could be caused by GWs generated at the solar terminator. Xu et al. (2015) once used six all-sky imagers and satellite sensors from Fengyun-2 (FY-2) and reported obvious concentric gravity wave events in the China region, which is associated with strong 
thunderstorms. Figueiredo et al. (2018) suggested that AGWs could play an important role in generating MSTIDs. Oinats et al. (2016) found that the relative amplitude of MSTIDs increased with an increase in solar activity, but the effect of geomagnetic activity on MSTIDs remained unclear.

Kelley and Miller (1997) found that the MSTID characteristics were not always consistent with the classic theory of GWs. Plasma instability, e.g., Perkins instability, was considered another candidate mechanism for midlatitude nighttime MSTIDs (Perkins 1973). However, the growth rate associated with Perkins instability was too small to explain the observations of nighttime MSTIDs (Kelley and Makela 2001; Garcia et al. 2000). Cosgrove and Tsunoda (2002, 2004), Tsunoda and Cosgrove (2001) and Cosgrove et al. (2004) proposed a generation mechanism based on the coupling of the midlatitude $\mathrm{E}$ layer and F layer. Otsuka et al. (2007) also showed the interlink between the $\mathrm{E}$ region and $\mathrm{F}$ region with regard to MSTIDs during nighttime. The Es layer instability combined with the Perkins instability of the F layer provided a greater growth rate after coupling than either the Es layer instability or the $\mathrm{F}$ layer instability alone. In addition, GWs as a perturbation were suggested to accelerate the growth rate related to the Es layer and Perkins instability (Kelley and Makela 2001; Shiokawa et al. 2003; Makela et al. 2010; Yokoyama et al. 2009). Based on recent allsky imager and GNSS observations, Huang et al. (2016) also indicated that the nighttime MSTIDs in midlatitude China may be generated by the coupling of electrodynamic processes. Recently, Narayanan et al. (2018) showed that electrodynamic coupling is necessary for the formation of nighttime MSTIDs with conjugate observations of the $\mathrm{E}$ and $\mathrm{F}$ regions from both hemispheres.

To calculate the propagation characteristics of MSTIDs, local antenna arrays or distributed receivers were utilized by early researchers. Due to the low cost of maintenance, global positioning system (GPS) receiver networks with different base lengths were widely utilized to monitor the structure and distributions of MSTIDs (Saito et al. 1998; Ding et al. 2011; Kotake et al. 2007). Saito et al. (1998) presented two-dimensional maps of total electron content (TEC) perturbations showing MSTIDs over Japan by using a dense GPS network with a mean distance of $\sim 25 \mathrm{~km}$ between receivers. Ding et al. (2011) studied the climatology of MSTIDs in central China by using observations of GPS networks with average distances of $\sim 20-40 \mathrm{~km}$ between stations. Kotake et al. (2007) reported a statistical study of MSTIDs observed in southern California with a GPS network, and the distance between the two receivers was approximately $45 \mathrm{~km}$. Otsuka et al. (2013) analyzed the TEC data observed by more than 800 permanent GPS receivers in
Europe and reported the statistical characteristics of the MSTIDs over Europe in 2008.

The present work focuses on the statistical analysis of MSTIDs using the Hong Kong Satellite Positioning Reference Station Network (SatRef). The average baseline length of SatRef is approximately $10-15 \mathrm{~km}$, which is shorter than that in previous studies. The observed MSTIDs are categorized and analyzed according to LT, season, geomagnetic activity and tropical cyclone. We discuss the limitations and conditions of short baseline GNSS measurements. The potential sources of MSTIDs observed in this region are also discussed.

\section{Data and method}

The Hong Kong Satellite Positioning Reference Station Network (SatRef) provides RINEX data from 2014 to 2017 obtained from dual-frequency GPS receivers. Figure 1 shows geographical locations of the receivers in Hong Kong $\left(22^{\circ} 15^{\prime} \mathrm{N}, 114^{\circ} 15^{\prime} \mathrm{E}\right)$, which was acquired from the Survey and Mapping Office/Lands Department in Hong Kong (http://www.geodetic.gov.hk/smo/gb/downv .aspx). The total number of GPS receivers is 18 (T430 replaced HKFN since 4 March, 2015), and the average distance between any two receivers is approximately 10-15 km. In previous studies, the distance between receivers was approximately $15-45 \mathrm{~km}$. The distribution of such intensive stations provides effective information for TEC measurements.

GPS satellites transmit L-band carriers of two frequencies to the ground ( $\mathrm{f} 1=1.57542 \mathrm{GHz}, \mathrm{f} 2=1.22760 \mathrm{GHz}$ ), and the observation stations record the carrier phase every $30 \mathrm{~s}$. Slant TEC (STEC) is calculated from the carrier phase leveling or pseudorange smoothing techniques. Slant TEC can be represented as a function of

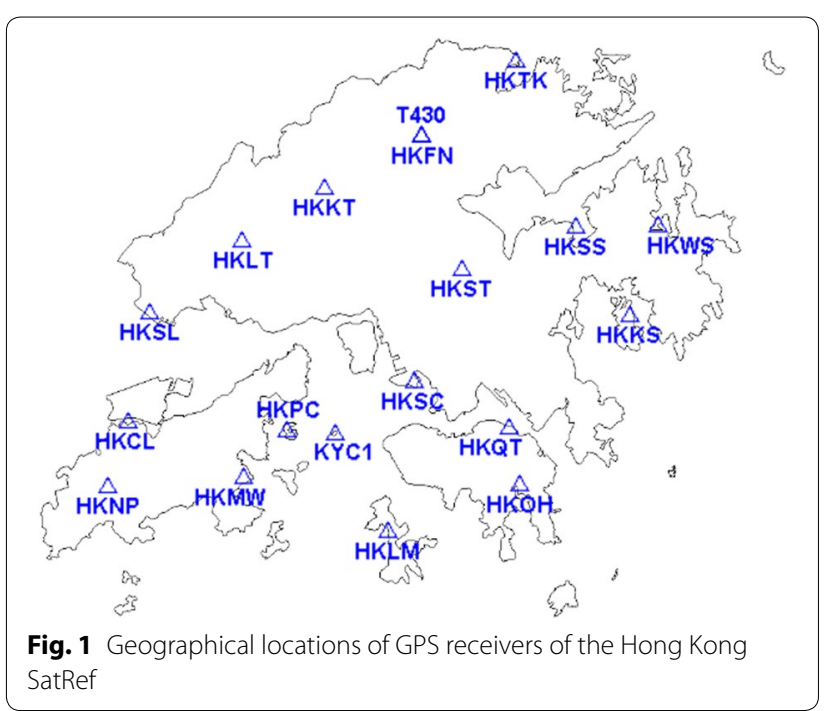


time $t$ and the angle $z$ between the line of sight (LOS) and the local zenith at its ionospheric penetration point (IPP) using the following equation (Ozeki and Heki 2010):

$$
\mathrm{STEC}=\operatorname{VTEC}(t) / \cos z+d
$$

where VTEC is the TEC when the LOS is perpendicular to the ionosphere, and $d$, which is the pseudorange bias, remains constant for each satellite and receiver during the study period. After a long period of observation, the VTEC calculation is free from $d$ because $z$ changes with time when the satellites move in orbit and $z$ can be calculated from the GPS satellite orbital data. The VTEC variation can be approximately modeled by the quadratic function of $t$ within a 1.2-h time series by the following equation:

$$
\operatorname{VTEC}(t)=a t^{2}+b t+c
$$

We estimate the values of $a, b, c$, and $d$ by the least squares method. STEC deviation (DSTEC), which can describe the TEC perturbation intensity, is derived from the difference between the observed STEC and the STEC calculated by the model.

We select GPS observations with elevation angles larger than $60^{\circ}$ by considering multipath effects, which also focus the observations on the ionospheric region around Hong Kong. After calculating the STEC, the multichannel maximum entropy method (MMEM) is used to analyze the TEC data observed from several GPS stations and obtain the propagation parameters of MSTIDs. MMEM (Ding et al. 2011; Huang et al. 2016) requires that the TEC data are obtained from three non-collinear GPS stations for the cross-spectral analysis (Ding et al. 2004, 2011). The MMEM details in our study are discussed in "The method to obtain the propagation parameters of MSTIDs" section. For the short baseline measurements of the CORS network in our study, it is necessary to consider whether the phase difference of the MSTID can be extracted from the TEC data. We further discuss the feasibility of MSTID observations by using short baseline measurements in "Short baseline measurements of the CORS network' section.

\section{The method to obtain the propagation parameters of MSTIDs}

STEC time series of different satellites can be simultaneously observed from a single station with different tracks of IPPs. Jacobson et al. (1995) noted that when a TID passes by, the TEC perturbations can be observed in more than one slant TEC series with different perturbation amplitudes. For each station, we select a TEC time series with the largest perturbation amplitude for subsequent data processing to find the most important TID characteristics. Three non-collinear CORS stations correspond to three STEC time series.

The raw STEC time series were observed simultaneously from three non-collinear CORS stations. We used a sliding window with a length of $1.2 \mathrm{~h}$ and a time interval of 2 min to process the raw STEC series. In this case, each obtained time series contained 144 data points, and there are 4 data points between two adjacent time series due to the $30 \mathrm{~s}$ time resolution of the GPS data. The background TEC, i.e., the running averaged TEC from each station, is obtained from the least polynomial fits, and we calculated the DSTEC by subtracting the background TEC. In our study, the MMEM (Ding et al. 2011; Huang et al. 2016) utilized three DSTECs for the cross-spectral analysis, so the main frequency of the waves and phase difference between two stations were derived. Due to the movement of IPPs, there is an offset up to $30 \%$ in the main frequency of MSTIDs (Ding et al. 2011), which is similar to the Doppler shift. To remove the IPP movement effect, a Galileo transform (Wan et al. 1997) was used to adjust the main frequency.

Based on the adjusted main frequency and phase difference, we calculated the fluctuation period, azimuth angle, phase velocity and wavelength using the equation given by Wang et al. (2007) and Ding et al. (2004). Since each time interval between two time series of $1.2 \mathrm{~h}$ was $2 \mathrm{~min}$, the propagation parameters can be seen as a function of time during the observed time. The uncertainties in the estimated period, phase velocity, and azimuth using MMEM are approximately $25 \%, 40 \%$ and $20 \%$, respectively (Ding et al. 2011; Huang et al. 2016). When a steady TID field passes by, the propagation parameters will remain constant for the duration of the field. Three conditions are required to determine whether an MSTID event occurs. First, the maximum amplitudes of disturbances should exceed 1 TECU (1 TECU $=10^{16}$ electrons $/ \mathrm{m}^{2}$ ). Second, wavelike variations in the TEC series should last at least two cycles, which means that the DSTEC value should exceed 1 TECU at least twice during our observation. Third, the differences in the two sets of results for the period, azimuth, wavelength and phase velocity obtained by MMEM should be less than $10 \mathrm{~min}$, $15^{\circ}, 24 \mathrm{~km}$ and $40 \mathrm{~m} / \mathrm{s}$, respectively. These three conditions ensure that we observe a steady wave, and thus, the obtained parameters are reliable.

\section{Short baseline measurements of the CORS network}

TEC in the ionosphere has been widely studied in recent years using GPS networks. To obtain accurate and effective information for TEC measurements, it is necessary to establish a GPS network with sufficient stations. The distance between two receivers was generally approximately $15-45 \mathrm{~km}$ in previous studies (Saito et al. 1998; 
Ding et al. 2011; Kotake et al. 2007). In this study, receivers with a baseline of $10-15 \mathrm{~km}$ are utilized. For this short baseline measurement of the urban CORS network, we need to consider whether the distances between the three chosen stations for MMEM satisfy the requirement, that is, whether the phase difference of the MSTID can be extracted from the TEC data for analysis. Wavelike ionospheric perturbations can be distinguished if the following two conditions are satisfied.

1. At the height of the ionospheric piercing points, approximately $350 \mathrm{~km}$ from the ground, the distance s1 between the IPPs of the same satellite corresponding to different GPS stations should be shorter than the MSTID wavelength; that is, the phase difference extracted from different GPS stations should be less than $2 \pi$; otherwise, the recovered wave will be blurred, and a calculation error will be generated for the MMEM method. By knowing the position of the observation stations and their ground distances, s1 can be obtained from the IPP height and the elevation and azimuth of the satellite.

2. The satellites record data every $30 \mathrm{~s}$ and transmit these data to several observation stations. The distance between any two observation stations must be longer than the projection of the GPS satellite moving distance within $30 \mathrm{~s}$ at the ionosphere height; that is, s2, which is the minimum distance of the IPPs of any two stations receiving a signal from the same satellite, must be longer than $\mathrm{s} 3$, which is the maximum moving distance of the IPPs in all satellites received by the same station within $30 \mathrm{~s}$. In this case, the samplings are so accurate that the recovered TEC perturbations could be more reliable. Otherwise, the results of the cross-spectrum analysis are meaningless because the satellite signals received by the two receivers are exactly the same.

As seen in Fig. 1, the distance between HKNP $\left(22^{\circ} 15^{\prime} \mathrm{N}\right.$, $\left.113^{\circ} 53^{\prime} \mathrm{E}\right)$ and HKWS $\left(22^{\circ} 26^{\prime} \mathrm{N}, 114^{\circ} 20^{\prime} \mathrm{E}\right)$ is the farthest at approximately $49.74 \mathrm{~km}$. After calculation and comparison, s1 is approximately $48.5 \mathrm{~km}$, and the MSTID wavelength in this study is approximately $200-600 \mathrm{~km}$. Therefore, condition (1) is satisfied. From Fig. 1, the distance between HKPC $\left(22^{\circ} 17^{\prime} \mathrm{N}, 114^{\circ} 02^{\prime} \mathrm{E}\right)$ and $\mathrm{KYC} 1$ $\left(22^{\circ} 17^{\prime} \mathrm{N}, 114^{\circ} 04^{\prime} \mathrm{E}\right)$ is the shortest at approximately $3.95 \mathrm{~km}$, which means that the distance between the IPPs of the two stations receiving a signal from the same satellite is the shortest. We collected all $30 \mathrm{~s}$ of the data from the two stations receiving PRN14 from 20:00 to 22:00 UT on July 7, 2017. The statistical distribution of the IPP moving distances is shown in Fig. 2a, and s2 is approximately $3.82 \mathrm{~km}$. Then, we counted all the statistical distributions of IPP moving distances received by the HKQT station within 30 s on July 7, 2017, as shown in Fig. 2b, and s3 is approximately $2.1 \mathrm{~km}$. As a result, s2 is longer than $\mathrm{s} 3$, and condition (2) is also satisfied.

Consequently, it is reasonable to study the MSTIDs by using this relatively short baseline measurement of the CORS network.

\section{Results}

We analyzed TEC data obtained by continuous observations from the Hong Kong CORS network from 2014 to 2017. To investigate the seasonal variation in the MSTID characteristics, the one-year interval is divided into four seasons: spring (February-April), summer (May-July), autumn (August-October), and winter
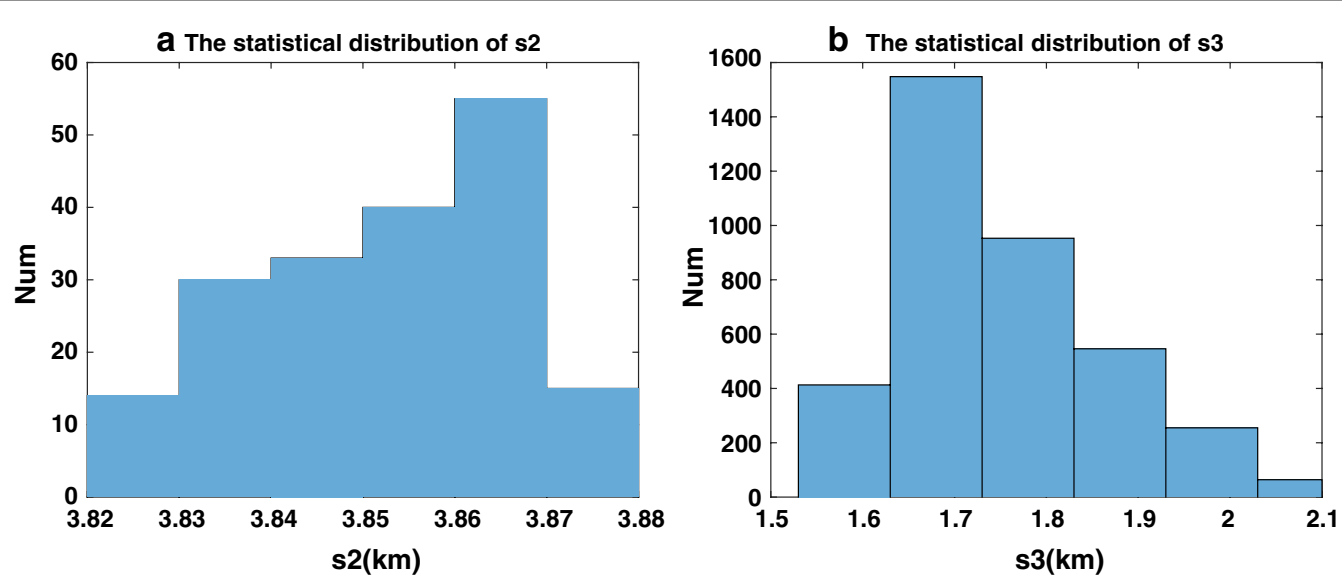

Fig. 2 a The statistical distribution of minimum IPP moving distances between HKPC and KYC1 for the ionosphere height on July 7, 2017. b The statistical distribution of IPP moving distances received by HKQT from PRN14 on July 7, 2017 
(November-January). Equinoxes are defined as 3 months from the centers of spring and autumn, and solstices are defined as 3 months from the centers of summer and winter.

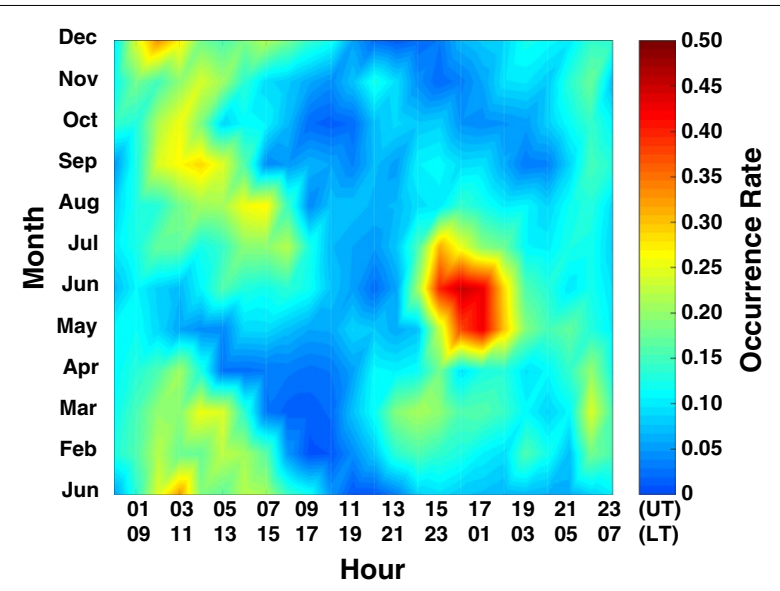

Fig. 3 LT and seasonal variations in MSTID occurrence rate in Hong Kong from 2014 to 2017
Figure 3 shows the LT and seasonal variation in the MSTID occurrence rate in month-hour bins. Here, the occurrence rate is defined as the ratio of the number of disturbances observed to the monthly and hourly total number of observations. In the figure, the MSTID occurrence rate was high during the daytime (1000-1700 local time (LT)) in spring, autumn and winter, during the nighttime (2200-0300 LT) in summer and spring, and during the morning (0500-0700 LT) in spring and autumn. The occurrence rate in the summer at midnight was the highest and exceeded $45 \%$, which was nearly twice that in other cases. This result is consistent with those of previous studies (Kotake et al. 2007; Figueiredo et al. 2018).

Figure 4 shows the relationship between LT and the occurrence rate of the propagation parameters for the MSTIDs in spring, summer, autumn and winter, including the period, horizontal wavelength and phase velocity. The occurrence rates in each bin were defined as the ratio of the number of disturbances observed to the total number of observations.

In Fig. $4 \mathrm{a}-\mathrm{d}$, the occurrence rate was binned as a function of LT with an interval of $1 \mathrm{~h}$ and a period of MSTID

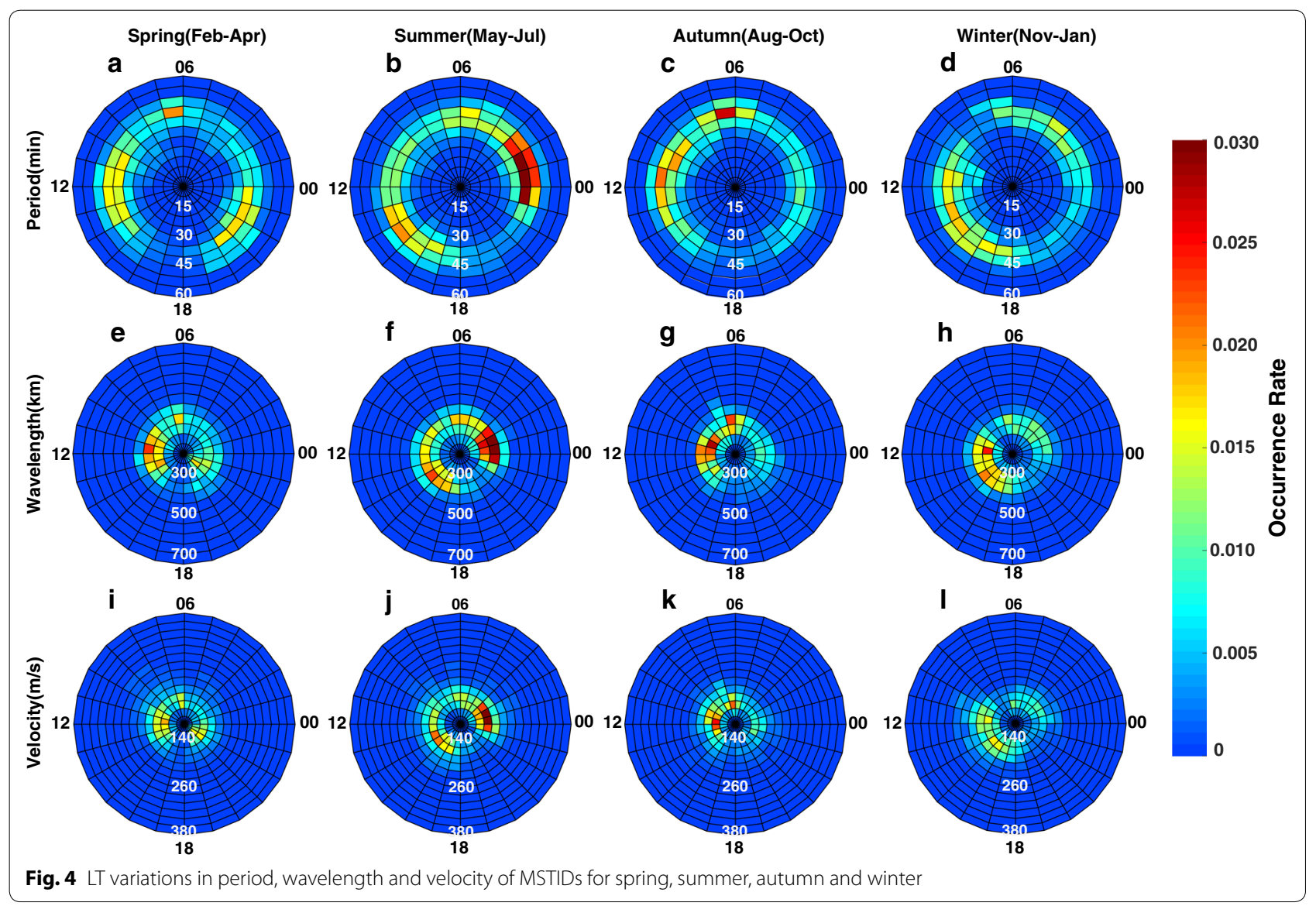


from 5 to $60 \mathrm{~min}$ in $5 \mathrm{~min}$ steps. The period of daytime MSTID (from 1000 to $1700 \mathrm{LT}$ ) in spring, autumn and winter was distributed between 20 and $50 \mathrm{~min}$ and mainly between 30 and 45 min in the spring and summer at nighttime (from 2200 to $0300 \mathrm{LT}$ ). In some cases, the range of the MSTID period tended to be longer during the daytime than during the nighttime. The period of MSTID observed in the spring and autumn morning (from 0500 to $0700 \mathrm{LT}$ ) was mainly distributed between 35 and 50 min, as shown in Fig. 4a, c.

Figure $4 \mathrm{e}-\mathrm{h}$ shows the LT variation in the horizontal wavelength of the MSTIDs for spring, summer, autumn and winter. The data were divided into $50 \mathrm{~km}$ bins. The horizontal wavelength data in the spring, autumn and winter daytime (from 1000 to $1700 \mathrm{LT}$ ) were mainly distributed between 250 and $450 \mathrm{~km}$, while these data were between 200 and $450 \mathrm{~km}$ in the spring and summer nighttime (from 2200 to 0300 LT). In some cases, the horizontal wavelength tended to be longer during daytime than nighttime. The horizontal wavelength data in the spring and autumn morning (from 0500 to 0700
LT) were widely distributed between 300 and $450 \mathrm{~km}$, as shown in Fig. 4e-g.

Figure $4 \mathrm{i}-\mathrm{l}$ shows the $\mathrm{LT}$ variation in the phase velocity of MSTIDs for different seasons. The data were divided into $20 \mathrm{~m} / \mathrm{s}$ bins. Most phase velocity data were distributed between 140 and $240 \mathrm{~m} / \mathrm{s}$ in the spring, autumn and winter daytime (from 1000 to $1700 \mathrm{LT}$ ), whereas these data were between 120 and $220 \mathrm{~m} / \mathrm{s}$ in the spring and summer nighttime (from 2200 to $0300 \mathrm{LT}$ ). Comparing the results, the phase velocity was found to be slightly slower during nighttime than daytime. As shown in Fig. $4 \mathrm{i}, \mathrm{k}$, the phase velocity data during the spring and autumn mornings (from 0500 to $0700 \mathrm{LT}$ ) were widely distributed between 120 and $220 \mathrm{~m} / \mathrm{s}$.

In Fig. $5 \mathrm{a}-\mathrm{d}$, the polar plots with angles around the circumference of a circle representing azimuth (north at the top and 0 degrees, east at right and 90 degrees), and the concentric circles presented the LT. The occurrence rate was binned as a function of LT with an interval of $1 \mathrm{~h}$ and the MSTID azimuth was from $0^{\circ}$ to $360^{\circ}$ in steps of $30^{\circ}$. The figure shows the same LT variation as

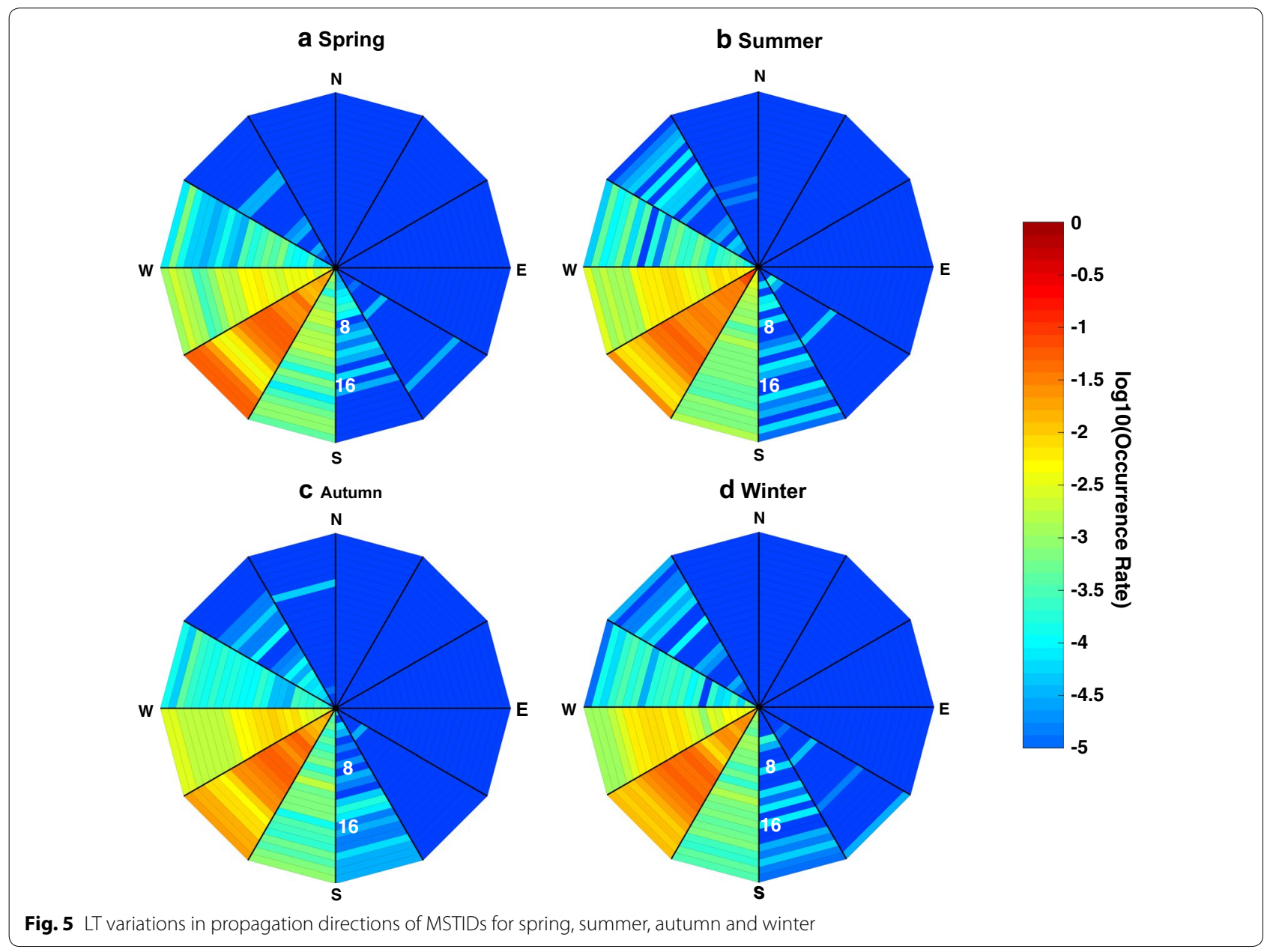


Fig. 3. The relatively high occurrence rate can be seen at 1000-1700 LT, 2200-0300 LT and 0500-0700 LT. In the summer and autumn, when cyclone events from the western Pacific are frequent around Hong Kong, the MSTIDs are more often propagated northwestward than in spring and winter, as shown in Fig. 5b, c. The azimuth data in the spring, autumn and winter daytimes were mainly distributed between $180^{\circ}$ and $270^{\circ}$, while in the nighttimes in spring and summer, the data were mainly between $210^{\circ}$ and $300^{\circ}$. In Fig. $5 \mathrm{a}$, c, the azimuth data in the spring and autumn mornings were widely distributed between $210^{\circ}$ and $270^{\circ}$. The propagation direction of MSTID in all seasons tends to be southwest-west.

We found that MSTIDs during 2014-2017 can be categorized into three types. One type is daytime MSTIDs, which frequently occurred in spring, autumn and winter. The azimuth, period, phase velocity and horizontal wavelength of daytime MSTIDs were $180^{\circ}-270^{\circ}, 20-50 \mathrm{~min}$, $140-240 \mathrm{~m} / \mathrm{s}$ and $250-450 \mathrm{~km}$, respectively. The second type is nighttime MSTIDs, which mainly occurred in spring and summer. The four propagation parameters of nighttime MSTIDs were $210^{\circ}-300^{\circ}, 30-45 \mathrm{~min}, 120-$ $220 \mathrm{~m} / \mathrm{s}$ and $200-450 \mathrm{~km}$, respectively. The third type is morning MSTIDs, which mainly occurred in spring and autumn, and the four propagation parameters were $210^{\circ}-270^{\circ}, 35-50 \mathrm{~min}, 120-220 \mathrm{~m} / \mathrm{s}$ and $300-450 \mathrm{~km}$, respectively. The wavelength and phase velocity of daytime MSTIDs seem greater than those of the nighttime MSTIDs.

To investigate the dependence of the MSTID occurrence rates on geomagnetic activity, the data in Fig. 6 were categorized into three sets of $0 \leq \mathrm{Kp}<3,3 \leq \mathrm{Kp}<6$, and $6 \leq \mathrm{Kp}<9$ to represent the quiet, moderate and active conditions of geomagnetic activity, respectively. Figure 6 a shows the total observation number with available data as a function of the LT distribution under different geomagnetic conditions. Since the Kp index is the same every $3 \mathrm{~h}$, the total number of observations every $3 \mathrm{~h}$ is the same under geomagnetic conditions. For example, the total number of observations at 0000, 0001 and 0002 UT was 1050 under the quiet geomagnetic condition in Fig. 6a. Figure $6 \mathrm{~b}$ shows the MSTID occurrence rate in the LT distributions under different geomagnetic conditions. For the geomagnetically quiet and moderate conditions, $0 \leq \mathrm{Kp}<3$ and $3 \leq \mathrm{Kp}<6$, the occurrence rates with a maximum of 0.1802 (quiet condition) and 0.2323 (moderate condition) were relatively low and presented three peaks (approximately 1100, 0100 and 0600 LT) in the local time distribution. However, for the geomagnetically active times, $6 \leq \mathrm{Kp}<9$, the occurrence rate was as high as 0.3014 at $2200 \mathrm{LT}$. In general, intense geomagnetic activity shows a higher occurrence rate, while due to a lower number of observation hours, the LT variation under geomagnetically active conditions is unclear.

In each subplot of Fig. 7, the occurrence rate of MSTIDs was binned as a function of LT and propagation parameters. The occurrence rate in each bin was calculated as the ratio of the number of disturbances observed to the total number of observations in the corresponding category. For the geomagnetically quiet and moderate conditions, the occurrence rate was much lower than that for the active time. Under active geomagnetic conditions, the azimuth, period, phase velocity and horizontal wavelength of the MSTIDs were $220^{\circ}-260^{\circ}, 30-50 \mathrm{~min}$, $250-450 \mathrm{~km}$ and $120-200 \mathrm{~m} / \mathrm{s}$, respectively.

Vadas and Fritts (2009) proposed that the GWs propagated upward as concentric rings from the convection plume, and the radius of the concentric circle simultaneously increased with the enhancement in height. Vadas (2007) suggested that if GWs from a tropospheric source propagate into the thermosphere, the corresponding MSTIDs will not be observed more than $\sim 2000 \mathrm{~km}$ from this source. To investigate the dependence of the MSTID occurrence rates on AGWs in relation to tropical cyclones, all 67 tropical cyclone events whose centers were located less than $2000 \mathrm{~km}$ away from Hong Kong were collected, which occurred mainly in summer, autumn, winter and a small number occurred in spring, including tropical storm, typhoon and tropical depression from January 2014 to December 2017 in the Asia-Pacific region according to the typhoon database (http://typhoon.zjwater.gov.cn/default.aspx?tdsourceta $\mathrm{g}=$ s_pcqq_aiomsg).

We calculated the total occurrence percentage of MSTIDs during the cyclone period and the normal period of daytime (1000-1700 LT) and nighttime (2200-0300 LT), which was approximately $25.22 \%$ (21.88\%) during the cyclone period (normal period) in the daytime and approximately $20.79 \%$ (21.37\%) in the nighttime. Figure 3 shows that the MSTID occurrence depends strongly on season and LT. Therefore, we discussed the influence of cyclone activities on MSTIDs based on solstices (May-Jul and Nov-Jan) and equinoxes (Feb-Apr and Aug-Oct) to eliminate seasonal bias. Solstice and equinox data were sorted into normal and cyclone period, respectively. Figure 8 shows the occurrence rate of MSTIDs (Fig. 8a, b) and the observation number (Fig. 8c, d) in each hour bin during solstices and equinoxes from 2014 to 2017 . There were 8033 (7012) and 1535 (1593) hours when the MSTIDs were observed during the solstices (equinoxes) in the normal period and cyclone period, respectively. As shown in Fig. 8a, the occurrence rate of MSTIDs did not show a significant difference between the cyclone period and the normal period during both daytime and nighttime. However, in Fig. 8b, the occurrence rate in the equinox daytime 

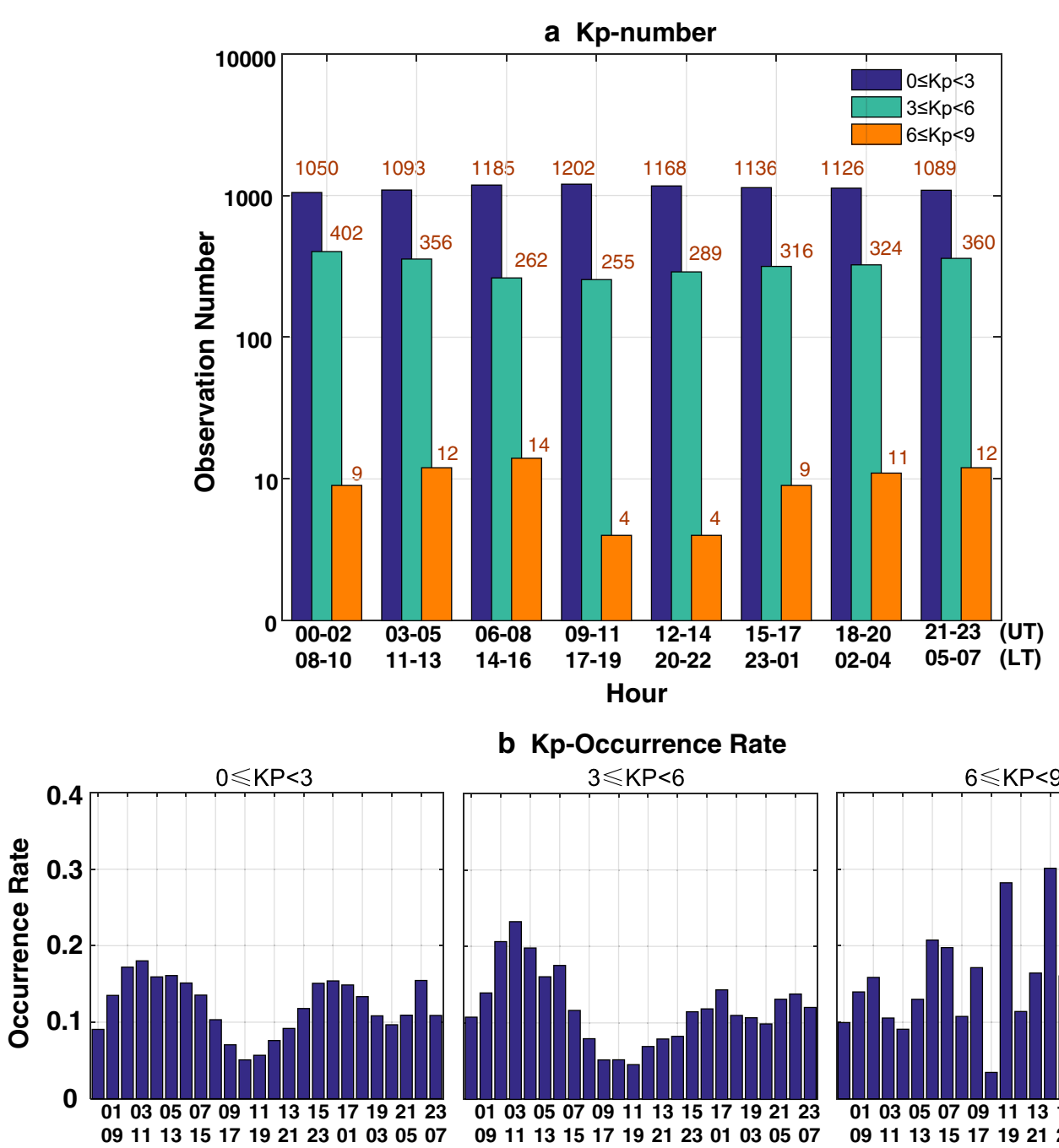

\section{b Kp-Occurrence Rate}
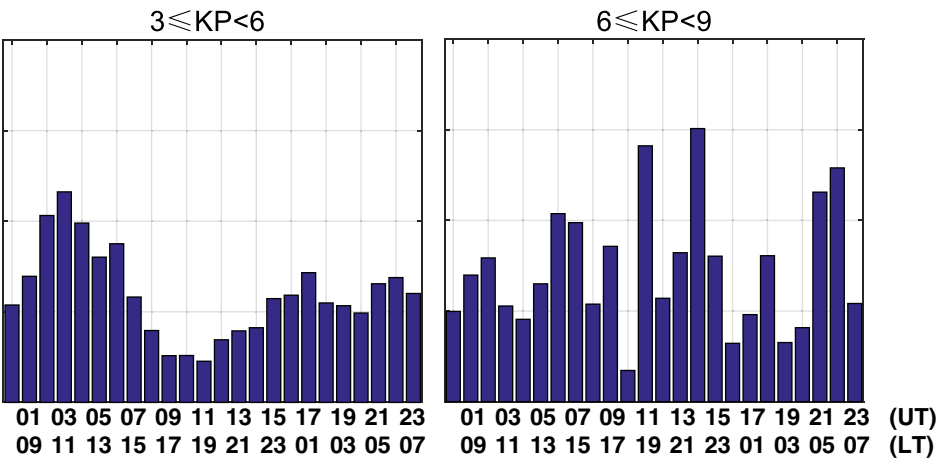

Hour

Fig. 6 The total observation number and occurrence rate of MSTIDs as a function of the LT distribution under different geomagnetic activity conditions

during the cyclone period was higher than that during the normal period, and the occurrence rate increased more than $10 \%$ at noon, while the occurrence rate in the nighttime during the cyclone period was slightly lower than that during the normal period.

In each subplot of Fig. 9, the occurrence rate of MSTIDs was binned as a function of LT and propagation parameters under the cyclone period. The azimuth, period, phase velocity and horizontal wavelength of MSTIDs were $240^{\circ}-300^{\circ}, 20-50 \mathrm{~min}, 200-450 \mathrm{~km}$ and $120-240 \mathrm{~m} / \mathrm{s}$, respectively.

\section{Discussion}

In the above analysis, we concentrated on the statistical distribution of MSTIDs observed in the low-latitude China region using Hong Kong SatRef data from 2014 to 2017. The MSTIDs were categorized and analyzed according to local time, season, geomagnetic activity and tropical cyclone. Our results are generally consistent with previous studies with various measurements in different places (Figueiredo et al. 2018; Jacobson et al. 1995; Kotake et al. 2007; Ding et al. 2011; Huang et al. 2016). However, some new findings, such as the potential 


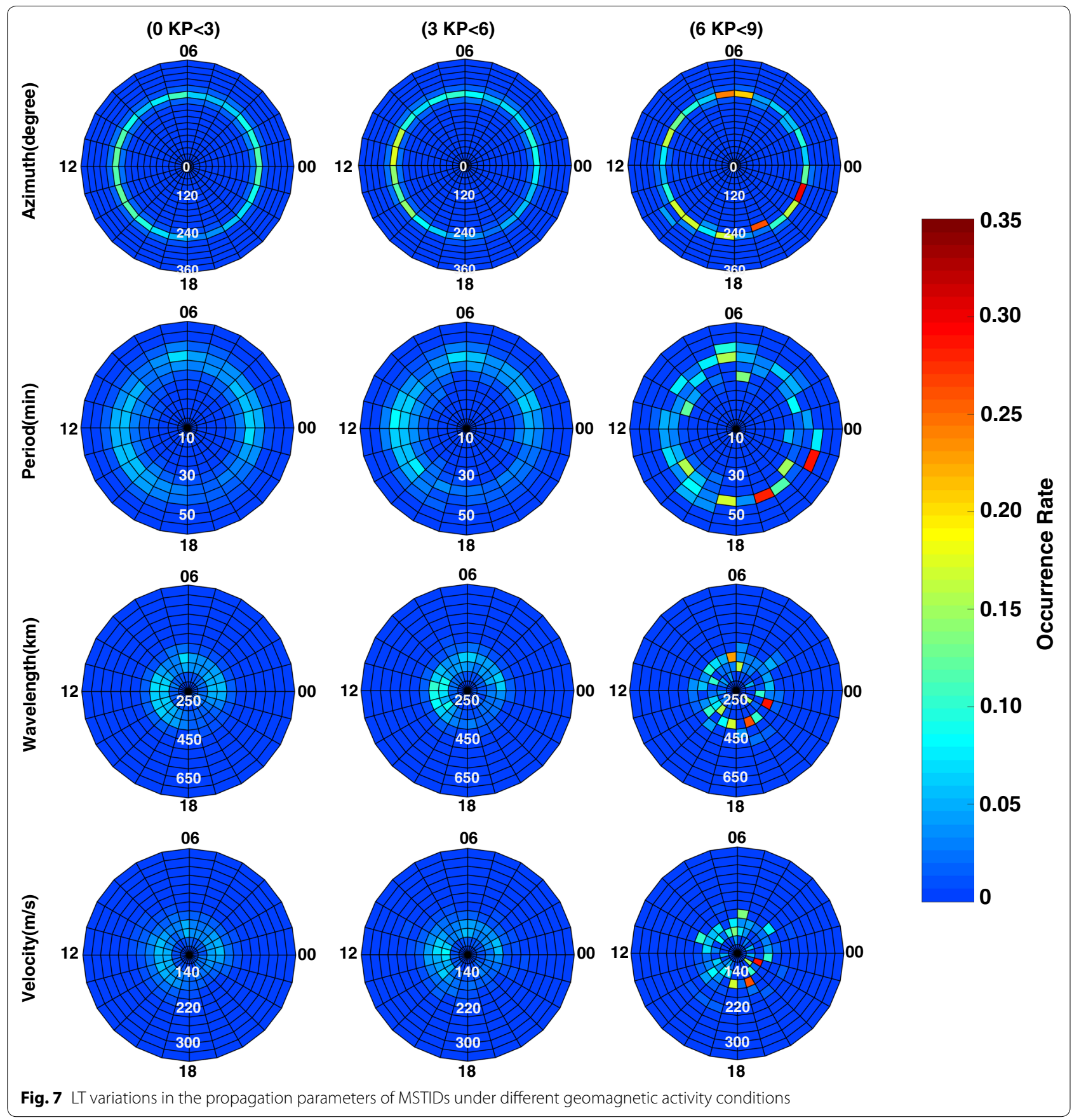

sources of MSTIDs observed in this region, are worth further discussion.

AGW have been thought to be one source of MSTIDs (Hines 1960; Hooke 1968). With the collision between neutral particles and charged particles, the energy and momentum of the AGW disturbance are transmitted to the charged particles, and the TID is thereby excited (Miller et al. 1997; Kelley and Miller 1997). Kotake et al. (2007) discussed the impact of this process on daytime
MSTIDs in detail. Recent studies by Vadas and Liu (2009) and Vadas and Crowley (2010) also indicated that the propagation direction of the TID generated by AGWs has no regularity and needs further research.

Previous studies found that nighttime MSTIDs propagate southwestward in the northern hemisphere and northwest in the southern hemisphere (Garcia et al. 2000; Shiokawa et al. 2003; Otsuka et al. 2004; Kotake et al. 2007; Makela et al. 2010; Otsuka et al. 2013). Our 


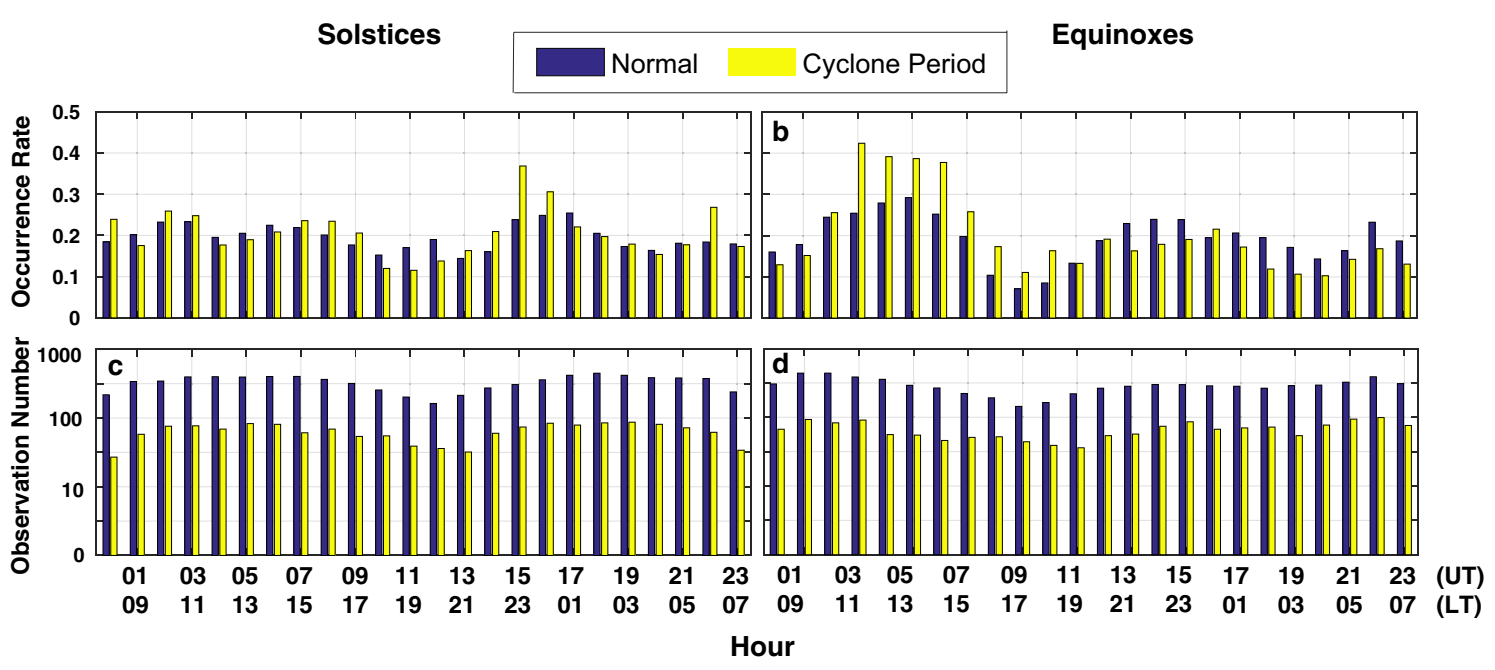

Fig. 8 LT variations in MSTID occurrence rates during solstices and equinoxes under different cyclone period conditions

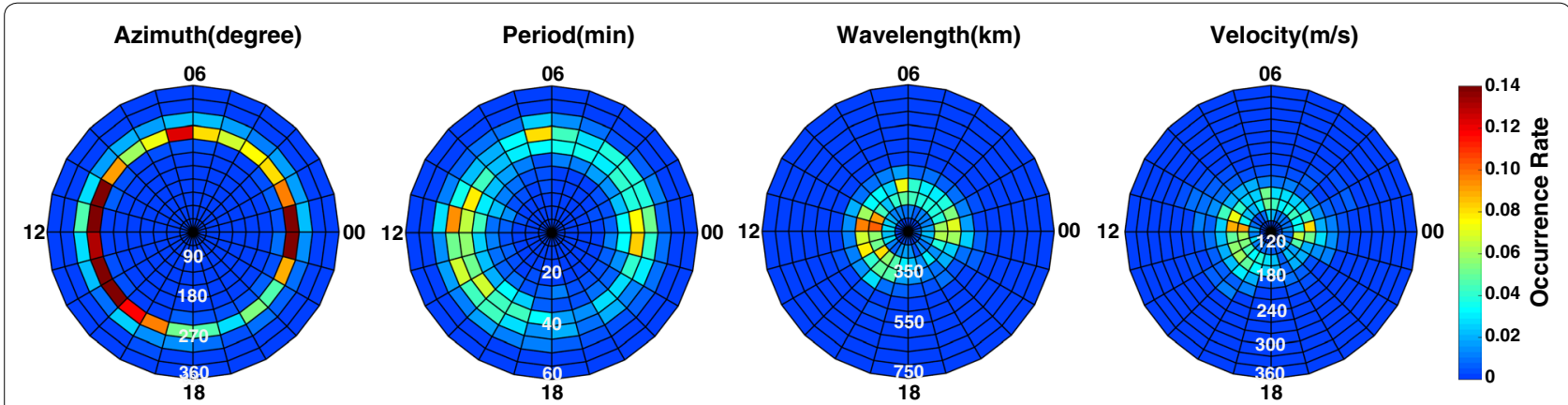

Fig. 9 LT variations in the propagation parameters of MSTIDs during the cyclone period

results are similar to those of this study. Figure 3 shows the maximum occurrence rate of nighttime MSTIDs during summer solstice. However, Lei et al. (2012) once reported that the neutral density shows a minimum at solstices, and GWs caused by a neutral atmosphere disturbance cannot explain this phenomenon. Perkins (1973) reported plasma instability, that is, Perkins instability, which is regarded as another mechanism of nighttime MSTIDs because it can correctly predict most of the phase front alignment observed during nighttime MSTIDs.

According to earlier statistical studies of large-scale TIDs (LSTIDs), the occurrence rates clearly grow with increasing geomagnetic activity (Tsugawa et al. 2004; Ding et al. 2008; Song et al. 2013, Oinats et al. 2016). From "Results" section, we know that intense geomagnetic activity shows a higher occurrence rate. According to Hunsucker (1982), one of the first identified sources of MSTIDs is auroral activity associated with geomagnetic activity. However, the relationship between the MSTIDs at low latitudes and geomagnetic activity requires further research.

GWs have various sources, including tornado, convection, typhoon and other atmospheric activities. Xiao et al. (2007) reported that MSTIDs in the ionosphere were always observed during the typhoon, especially a strong typhoon, landing on or near a mainland coast, which is one of the important ground sources of wavelike disturbances in the troposphere. Figueiredo et al. (2018) suggested that the possible source of daytime MSTIDs may be associated with convective activities. By using six allsky imagers and satellite sensors from Fengyun-2 (FY2), Xu et al. (2015) reported obvious concentric gravity wave events in the China region, which is associated with strong thunderstorms.

Due to the special geographical location, Hong Kong is often exposed to atmospheric activities such as tropical cyclones. In the daytime, the MSTIDs may be generated by GWs (Hines 1960; Hooke 1968; Hunsucker 1982; Kirchengast et al. 1996; Xiao et al. 2007; Kotake et al. 
2007; Figueiredo et al. 2018), whose sources include convection, typhoon and other atmospheric activities. The formation of nighttime MSTIDs is also closely related to electrodynamic coupling processes (Perkins 1973; Kelley and Makela 2001; Shiokawa et al. 2003; Cosgrove et al. 2004; Otsuka et al. 2007; Makela et al. 2010; Huang et al. 2016; Narayanan et al. 2018). Considering that the GWs propagate during the day and night, while the electrodynamic coupling processes generally occur only at night, the daytime MSTIDs are mainly generated by the GWs and the nighttime MSTIDs may be jointly generated by GWs and electrodynamic coupling processes. From Fig. 8b, the GWs caused by cyclones may contribute to the generation of daytime MSTIDs. At night, the occurrence rate during the cyclone period was slightly lower than that during the normal period, which may be related to the interaction between the GWs and electrodynamic coupling processes. We hypothesize that one of the possible mechanisms of MSTIDs may be associated with cyclones occurring during the observation duration. To examine this hypothesis, we collected the DSTEC value, which can describe the intensity of MSTIDs, and the calculated propagation parameters corresponding to different cyclone pressure values, which can describe the intensity of a cyclone.

In Fig. 10, the horizontal axis represents the different pressures of the cyclone, including tropical depression, tropical storm, severe tropical storm, typhoon, severe

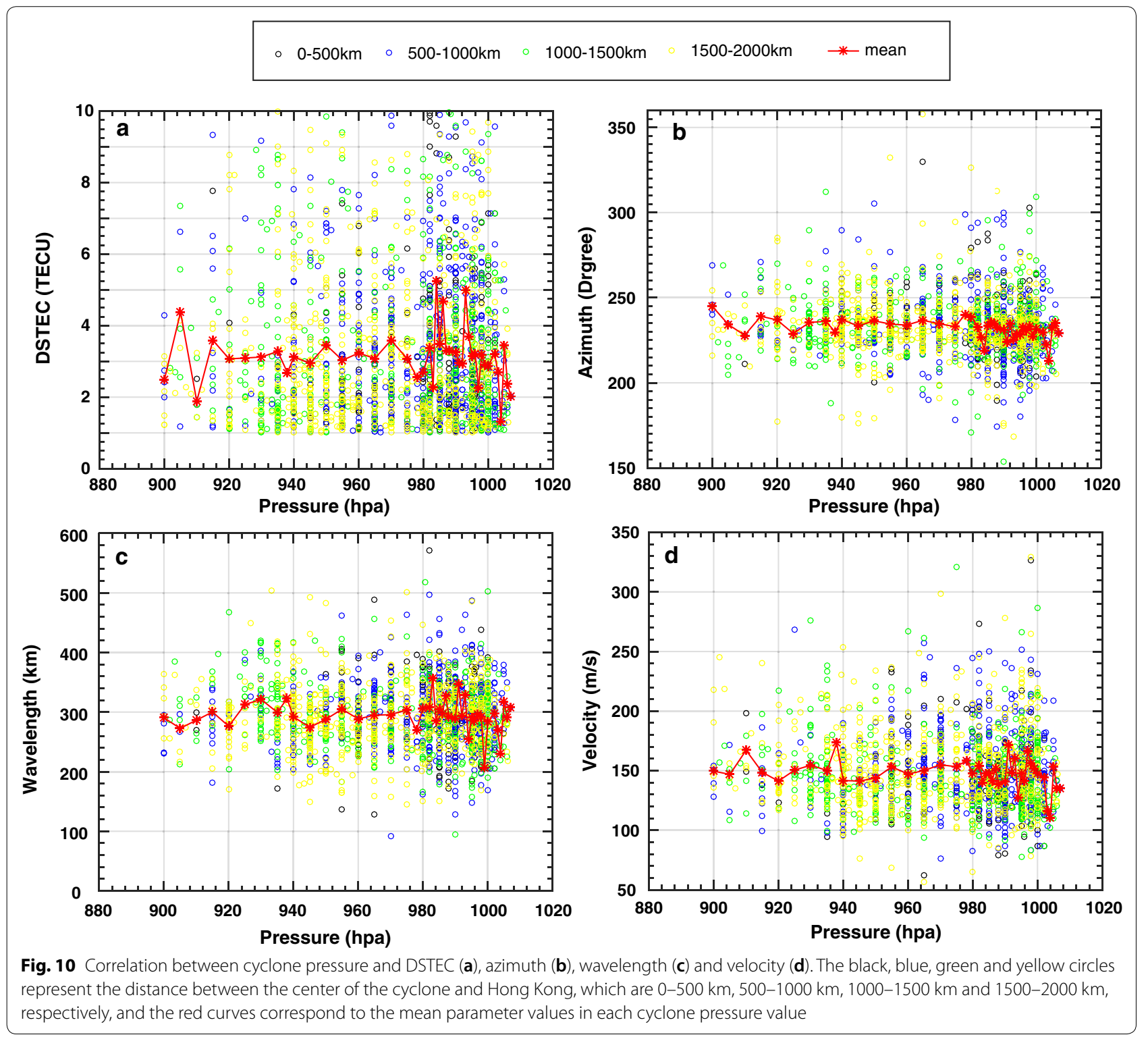


typhoon and super typhoon. The black, blue, green and yellow circles represent the distance between the center of the cyclone and Hong Kong, which are $0-500 \mathrm{~km}, 500-1000 \mathrm{~km}, 1000-1500 \mathrm{~km}$ and $1500-$ $2000 \mathrm{~km}$, respectively, and the number of observations was $213,602,514$ and 641 , respectively. The red curves correspond to the mean value of the parameters in each cyclone pressure value. In Fig. 10a, the mean DSTEC value varied significantly only when the pressure value was less than $920 \mathrm{hPa}$ and greater than $980 \mathrm{hPa}$. The mean MSTID azimuth did not show an obvious change from the cyclone pressure, as shown in Fig. 10b. In Fig. 10c, d, the mean values of wavelength and velocity show little change when the pressure value was less than $980 \mathrm{hPa}$, while these parameter values show relatively large fluctuations when the pressure value is greater than $980 \mathrm{hPa}$. However, given the uncertainties in the parameter estimations mentioned in "Data and method" section, the mean parameters did not seem to vary with the cyclone pressure, and we cannot deduce a significant difference in the MSTID parameters between the cyclone period and the normal period. The GWs excited by deep convection were dubbed 'primary GWs' by Vadas and Liu (2013), and the primary GW dissipation in the thermosphere can create secondary GWs. Since the MSTIDs in our study are determined whether the DSTEC is $>1$ TECU, it does not discriminate between primary and secondary GWs. Vadas and Liu (2013) also noted that while small- and medium-scale primary GWs from convective plumes can propagate only several hundred to several thousand kilometers, the secondary GWs created by the primary GWs can easily propagate tens of thousands of kilometers from these convective plumes. A possible reason for not finding noticeable differences between normal and cyclone activity is due to the $2000 \mathrm{~km}$ restriction for cyclone identification. The cyclone whose center was located more than $2000 \mathrm{~km}$ away from Hong Kong can generate primary GWs, and then, the primary GWs dissipated and generated the secondary GWs. These secondary GWs propagate farther than the primary GWs and reach over Hong Kong, causing the disturbance to the ionosphere and forming MSTIDs.

Combining Figs. 8, 9 and 10, the occurrence rate in the equinox daytime during the cyclone period was higher than that during the normal period, while due to the distance restriction for cyclone identification and different propagation distances of the primary GWs and secondary GWs, there is no clear difference in MSTID parameters during cyclone activity and normal period. However, further investigation is needed to clarify this finding in detail.

\section{Summary}

We have presented a statistical analysis of the occurrence rate of MSTIDs observed by the Hong Kong CORS network. Our statistical study is generally consistent with previous studies in the midlatitude region (Ding et al. 2011; Kotake et al. 2007; Figueiredo et al. 2018). However, there are several new findings in this study, which can be summarized as follows.

1. The occurrence rate of MSTIDs presents a strong dependence on LT and season. We found that MSTIDs during 2014-2017 can be categorized into three types. One type is daytime MSTIDs, frequently occurring at 1000-1700 LT in spring, autumn and winter. The second type is nighttime MSTIDs, mainly occurring at 2200-0300 LT in spring and summer. The third type is morning MSTIDs, mainly occurring at 0500-0700 LT in spring and autumn. The MSTID propagation characteristics are shown in "Results" section.

2. The propagation direction of nighttime MSTIDs is southwestward, which is consistent with the findings of previous studies (Kotake et al. 2007; Huang et al. 2016). However, the propagation directions of daytime MSTIDs are southwestward and westward, which is not consistent with the findings of previous studies (Kotake et al. 2007).

3. Under different geomagnetic conditions, the occurrence rate presents some differences, and intense geomagnetic activity shows a higher occurrence rate. However, due to the lower number of observation hours, the correlation between geomagnetic activity and the occurrence rate of MSTIDs in this region is not yet clear.

4. One possible reason for not finding noticeable differences in the MSTID parameter according to different cyclone activities may be the distance restriction for cyclone identification and different propagation distances of the primary GWs and secondary GWs.

5. MSTIDs can be investigated by using this short baseline measurement of an urban CORS network with a typical distance of approximately 10-15 km.

\section{Key Points}

- The occurrence rate of MSTIDs in Hong Kong during 2014-2017 shows strong dependence on local time and season.

- The MSTIDs can be observed by short baseline measurement. 
- MSTIDs in this region could be excited by various sources.

\begin{abstract}
Abbreviations
MSTID: medium-scale traveling ionospheric disturbance; CORS: continuously Operating Reference Stations; GW: gravity wave; MMEM: multichannel maximum entropy method; GPS: global positioning system; TEC: total electron content; AGW: atmospheric gravity wave; SatRef: Satellite Positioning Reference Station Network; GNSS: global navigation satellite system; LOS: line of sight; IPP: ionospheric penetration point; STEC deviation: DSTEC; LT: local time; TID: traveling ionospheric disturbance.
\end{abstract}

\section{Authors' contributions}

GC performed data processing and statistical analysis on TID and drafted the manuscript. CZ and YL elaborated on the processing and calculation of GPS data and supervised all the work of GC. JZ, QT, XW and ZZ participated in the discussion and interpretation of the statistical results obtained. All authors have read and approved the final manuscript.

\section{Acknowledgements}

The GPS data used in this work were supplied by Hong Kong Satellite Positioning Reference Station Network (SatRef) and Continuously Operating Reference Stations (CORS). The Kp data used in this work were supplied by ftp:// ftp.ngdc.noaa.gov/STP/GEOMAGNETIC_DATA/INDICES/KP_AP.This work was supported by the National Natural Science Foundation of China (NSFC grant No. 41574146, 41774162, and 41704155) and National Key R\&D Program of China (Grant No. 2018YFC15035XX).

\section{Competing interests}

The authors declare that they have no competing interests.

\section{Availability of data and materials}

The data that support the findings of this study are available upon request from the corresponding author.

\section{Consent for publication}

Not applicable.

\section{Ethics approval and consent to participate} Not applicable.

\section{Funding}

This work was supported by the National Natural Science Foundation of China (NSFC Grant No. 41574146, 41774162, and 41704155) and National Key R\&D Program of China (Grant No. 2018YFC15035XX).

\section{Publisher's Note}

Springer Nature remains neutral with regard to jurisdictional claims in published maps and institutional affiliations.

Received: 30 September 2018 Accepted: 23 April 2019

Published online: 02 May 2019

\section{References}

Bowman GG (1990) A review of some recent work on mid-latitude spread-

F occurrence as detected by ionosondes. J Geomagn Geoelectr 42:109-138

Cosgrove RB, Tsunoda RT (2002) A direction-dependent instability of sporadicE layers in the nighttime midlatitude ionosphere. Geophys Res Lett 29(18):1864. https://doi.org/10.1029/2002GL014669
Cosgrove RB, Tsunoda RT (2004) Instability of the E-F coupled nighttime midlatitude ionosphere. J Geophys Res 109:A04305. https://doi. org/10.1029/2003JA010243

Cosgrove RB, Tsunoda RT, Fukao S, Yamamoto M (2004) Coupling of the Perkins instability and the sporadic E layer instability derived from physical arguments. J Geophys Res 109:A06301. https://doi.org/10.1029/2003J A010295

Ding F, Yuan H, Wan W, Reid IM, Woithe JM (2004) Occurrence characteristics of medium-scale gravity waves observed in $\mathrm{OH}$ and $\mathrm{Ol}$ nightglow over

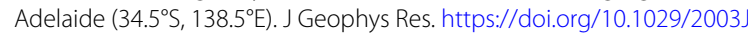
D004096

Ding F, Wan W, Liu L, Afraimovich EL, Voeykov SV, Perevalova NP (2008) A statistical study of large-scale traveling ionospheric disturbances observed by GPS TEC during major magnetic storms over the years 2003-2005. J Geophys Res. https://doi.org/10.1029/2008JA013037

Ding F, Wan W, Xu G, Yu T, Yang G, Wang J (2011) Climatology of medium-scale traveling ionospheric disturbances observed by a GPS network in central China. J Geophys Res. https://doi.org/10.1029/2011JA016545

Figueiredo CAOB, Takahashi H, Wrasse CM, Otsuka Y, Shiokawa K, Barros D (2018) Medium-scale traveling ionospheric disturbances observed by detrended total electron content maps over Brazil. J Geophys Res Space Phys 123:2215-2227. https://doi.org/10.1002/2017JA025021

Garcia FJ, Kelley MC, Makela JJ, Huang CS (2000) Airglow observations of mesoscale low velocity traveling ionospheric disturbances at midlatitudes. J Geophys Res 105:18407-18415. https://doi.org/10.1029/1999JA000305

Hines CO (1960) Internal atmospheric gravity waves at ionospheric heights. Can J Phys 38:1441-1481. https://doi.org/10.1139/p60-150

Hooke WH (1968) lonospheric irregularities produced by internal atmospheric gravity waves. J Atmos Terr Phys 30:795-823

Huang F, Dou X, Lei J, Lin J, Ding F, Zhong J (2016) Statistical analysis of nighttime medium-scale traveling ionospheric disturbances using airglow images and GPS observations over central China. J Geophys Res Space Phys 121:8887-8899. https://doi.org/10.1002/2016JA022760

Hunsucker RD (1982) Atmospheric gravity waves generated in the high latitude ionosphere: a review. Rev Geophys 20:293-315. https://doi. org/10.1029/RG020i002p00293

Jacobson AR, Carlos RC, Massey RS, Wu G (1995) Observation of traveling ionospheric disturbances with a satellite-beacon radio interferometer: seasonal and local time behavior. J Geophys Res 100(A2):1653-1665. https://doi.org/10.1029/94JA02663

Kelley MC, Makela JJ (2001) Resolution of the discrepancy between experiment and theory of midlatitude F-region structures. Geophys Res Lett 28:2589-2592. https://doi.org/10.1029/2000GL012777

Kelley MC, Miller CA (1997) Electrodynamics of midlatitude spread F, 3. Electrohydrodynamic waves? A new look at the role of electric fields in thermospheric wave dynamics. J Geophys Res 102:11539-11547. https:// doi.org/10.1029/96JA03841

Kirchengast G, Hocke K, Schlegel K (1996) The gravity wave-TID relationship: insight via theoretical model-EISCAT data comparison. J. Atmos. Sol. Terr. Phys. 58:233-243. https://doi.org/10.1016/0021-9169(95)00032-1

Klostermeyer J (1972) Numerical calculation of gravity wave propagation in a realistic thermosphere. J. Atmos. Sol. Terr. Phys. 34:765-774. https://doi. org/10.1016/0021-9169(72)90109-2

Kotake N, Otsuka Y, Ogawa T, Tsugawa T, Saito A (2007) Statistical study of medium-scale traveling ionospheric disturbances observed with the GPS networks in Southern California. Earth Planets Space 59:95-102. https:// doi.org/10.1186/BF03352681

Lei J, Matsuo T, Dou X, Sutton E, Luan X (2012) Annual and semiannual variations of thermospheric density: EOF analysis of CHAMP and GRACE data. J Geophys Res 117:A01310. https://doi.org/10.1029/2011JA017324

Makela JJ, Miller ES, Talaat ER (2010) Nighttime medium-scale traveling ionospheric disturbances at low geomagnetic latitudes. Geophys Res Lett 37:L24104. https://doi.org/10.1029/2010GL045922

Miller CA, Swartz WE, Kelley MC, Mendillo M, Nottingham D, Scali J, Reinisch B (1997) Electrodynamics of midlatitude spread F: 1. Observations of unstable, gravity waves-induced ionospheric electric fields at tropical latitudes. J Geophys Res 102:11521-11532. https://doi.org/10.1029/96JA03839

Morton FW, Essex EA (1978) Gravity wave observations at a southern hemisphere mid-latitude station using the total electron content technique. J Atmos Terr Phys 40:1113-1122. https://doi.org/10.1016/00219169(78)90059-4 
Narayanan VL, Shiokawa K, Otsuka Y, Neudegg D (2018) On the role of thermospheric winds and sporadic $E$ layers in the formation and evolution of electrified MSTIDs in geomagnetic conjugate regions. J Geophys Res Space Phys 123:6957-6980. https://doi.org/10.1029/2018JA025261

Oinats et al (2016) Statistical characteristics of medium-scale traveling ionospheric disturbances revealed from the Hokkaido East and Ekaterinburg HF radar data. Earth Planets Space 68:8. https://doi.org/10.1186/s4062 3-016-0390-8

Oliver WL, Otsuka Y, Sato M, Takami T, Fukao S (1997) A climatology of F region gravity wave propagation over the middle and upper atmosphere radar. J Geophys Res 102:14499-14512

Otsuka Y, Shiokawa K, Ogawa T, Wilkinson P (2004) Geomagnetic conjugate observations of medium-scale traveling ionospheric disturbances at midlatitude using all-sky airglow imagers. Geophys Res Lett 31:L15803. https://doi.org/10.1029/2004GL020262

Otsuka Y, Onoma F, Shiokawa K, Ogawa T, Yamamoto M, Fukao S (2007) Simultaneous observations of nighttime medium-scale traveling ionospheric disturbances and E region field-aligned irregularities at midlatitude. J Geophys Res. https://doi.org/10.1029/2005JA011548

Otsuka Y, Suzuki K, Nakagawa S, Nishioka M, Shiokawa K, Tsugawa T (2013) GPS observations of medium-scale traveling ionospheric disturbances over Europe. Ann Geophys 31:163-172. https://doi.org/10.5194/angeo $-31-163-2013$

Ozeki M, Heki K (2010) lonospheric holes made by ballistic missiles from North Korea detected with a Japanese dense GPS array. J Geophys Res 115:A09314. https://doi.org/10.1029/2010JA015531

Perkins F (1973) Spread F and ionospheric currents. J Geophys Res 78:218-226. https://doi.org/10.1029/JA078i001p00218

Saito A, Fukao S, Miyazaki S (1998) High resolution mapping of TEC perturbations with the GSI GPS network over Japan. Geophys Res Lett 25:30793082. https://doi.org/10.1029/98GL52361

Shiokawa K, Ihara C, Otsuka Y, Ogawa T (2003) Statistical study of nighttime medium-scale traveling ionospheric disturbances using midlatitude airglow images. J Geophys Res 108(A1):1052. https://doi.org/10.1029/2002J A009491

Song Q, Ding F, Wan WX et al (2013) Monitoring traveling ionospheric disturbances using the GPS network around China during the geomagnetic storm on 28 May 2011. Sci China: Earth Sci 56:718-726. https://doi. org/10.1007/s11430-012-4573-2

Tsugawa T, Saito A, Otsuka Y (2004) A statistical study of large-scale traveling ionospheric disturbances using the GPS network in Japan. J Geophys Res 109:6302. https://doi.org/10.1029/2003JA010302
Tsunoda RT, Cosgrove RB (2001) Coupled electrodynamics in the nighttime midlatitude ionosphere. Geophys Res Lett 28:4171-4174. https://doi. org/10.1029/2001GL013245

Vadas SL (2007) Horizontal and vertical propagation and dissipation of gravity waves in the thermosphere from lower atmospheric and thermospheric sources. J Geophys Res. https://doi.org/10.1029/2006JA011845

Vadas SL, Crowley G (2010) Sources of the traveling ionospheric disturbances observed by the ionospheric TIDDBIT sounder near Wallops Island on October 30, 2007. J Geophys Res 115:A07324. https://doi. org/10.1029/2009JA015053

Vadas SL, Fritts DC (2009) Reconstruction of the gravity wave field excited by convective plumes via ray tracing in real space. Ann Geophys 27:147-177

Vadas SL, Liu H (2009) Generation of large-scale gravity waves and neutral winds in the thermosphere from the dissipation of convectively generated gravity waves. J Geophys Res 114:A10310. https://doi. org/10.1029/2009JA014108

Vadas SL, Liu H-L (2013) Numerical modeling of the large-scale neutral and plasma responses to the body forces created by the dissipation of gravity waves from 6 h of deep convection in Brazil. J Geophys Res Space Phys 118:2593-2617. https://doi.org/10.1002/jgra.50249

Waldock JA, Jones TB (1986) HF Doppler observations of medium-scale travelling ionospheric disturbances at midlatitudes. J Atmos Terr Phys 48:245-260

Wan WX, Ning BQ, Yuan H, Li JN, Li L, Liang J (1997) TID observation using a short baseline network of GPS receivers. Acta Geodyn Geophys Hung 32(3-4):321-327

Wang M et al (2007) Monitoring global traveling ionospheric disturbances using the worldwide GPS network during the October 2003 storms. Earth Planets Space 59:1-13

Xiao Z, Xiao S, Hao Y, Zhang D (2007) Morphological features of ionospheric response to typhoon. J Geophys Res 112:A04304. https://doi. org/10.1029/2006JA011671

$\mathrm{Xu}$ J et al (2015) Concentric gravity waves over northern China observed by an airglow imager network and satellites. J Geophys Res Atmos 120:1105811078. https://doi.org/10.1002/2015JD023786

Yokoyama T, Hysell DL, Otsuka Y, Yamamoto M (2009) Three-dimensional simulation of the coupled Perkins and Es-layer instabilities in the nighttime midlatitude ionosphere. J Geophys Res 114:A03308. https://doi. org/10.1029/2008JA013789

\section{Submit your manuscript to a SpringerOpen ${ }^{\circ}$ journal and benefit from:}

- Convenient online submission

- Rigorous peer review

- Open access: articles freely available online

- High visibility within the field

Retaining the copyright to your article

Submit your next manuscript at springeropen.com 